\title{
Mediterranean Diet and Atherothrombosis Biomarkers: A Randomized Controlled Trial
}

\author{
Álvaro Hernáez,* Olga Castañer, Anna Tresserra-Rimbau, Xavier Pintó, Montserrat Fitó, \\ Rosa Casas, Miguel Ángel Martínez-González, Dolores Corella, Jordi Salas-Salvadó, \\ José Lapetra, Enrique Gómez-Gracia, Fernando Arós, Miquel Fiol, Lluis Serra-Majem, \\ Emilio Ros, and Ramón Estruch
}

Scope: To assess whether following a Mediterranean diet (MedDiet) improves atherothrombosis biomarkers in high cardiovascular risk individuals. Methods and results: In 358 random volunteers from the PREvención con Dleta MEDiterránea trial, the 1 -year effects on atherothrombosis markers of an intervention with MedDiet, enriched with virgin olive oil (MedDiet-VOO; $n$ $=120$ ) or nuts (MedDiet-Nuts; $n=119$ ) versus a low-fat control diet $(n=$ 119 ), and whether large increments in MedDiet adherence ( $\geq 3$ score points, versus compliance decreases) and intake changes in key food items are associated with 1-year differences in biomarkers. Differences are observed between 1-year changes in the MedDiet-VOO intervention and control diet on the activity of platelet activating factor acetylhydrolase in high-density lipoproteins (HDLs) (+7.5\% [95\% confidence interval: 0.17; 14.8]) and HDL-bound $\alpha_{1}$-antitrypsin levels $(-6.1 \%$ [-11.8; -0.29$\left.]\right)$, and between the MedDiet-Nuts intervention and the control arm on non-esterified fatty acid concentrations $(-9.3 \%[-18.1 ;-0.53])$. Large MedDiet adherence increments are associated with less fibrinogen $(-9.5 \%[-18.3 ;-0.60])$ and non-esterified fatty acid concentrations $(-16.7 \%[-31.7 ;-1.74])$. Increases in nut, fruit, vegetable, and fatty fish consumption, and decreases in processed meat intake are linked to enhancements in biomarkers.

Conclusion: MedDiet improves atherothrombosis biomarkers in high cardiovascular risk individuals.

\section{Introduction}

Better adherence to a traditional Mediterranean diet (MedDiet) prevents major cardiovascular clinical outcomes. ${ }^{[1-3]}$ These beneficial effects may be mediated by improvements in risk factors related to glucose metabolism, endothelial function, lipid profile, oxidative stress, and low-grade inflammation. ${ }^{[4]}$ Nevertheless, little evidence related to MedDiet effects on atherothrombosis mechanisms is available, although the antithrombotic properties of some of its individual components are known. ${ }^{[5,6]}$

Increased levels of biomarkers of platelet aggregation (P-selectin, ${ }^{[7]}$ platelet factor- $4^{[8]}$ ) and coagulation (fibrinogen, ${ }^{[9]}$ prothrombin fragment $1+2$ (proportional to thrombin formation), ${ }^{[10]}$ antithrombin ${ }^{[11]}$, and decreased concentrations of fibrinolysis indicators (plasminogen activator inhibitor-1 -PAI$1-,{ }^{[12]} \mathrm{D}$-dimer ${ }^{[13]}$ ) have been linked to increased incidence of major atherosclerotic events in prospective human studies. In addition, high levels of
Dr. Á. Hernáez, Dr. R. Casas, Dr. E. Ros, Dr. R. Estruch

Cardiovascular Risk, Nutrition and Aging Research Unit

August Pi i Sunyer Biomedical Research Institute (IDIBAPS)

Barcelona 08003, Spain

E-mail: alvaro.hernaez1@gmail.com

Dr. Á. Hernáez, Dr. O. Castañer, Dr. A. Tresserra-Rimbau, Dr. X. Pintó,

Dr. M. Fitó, Dr. R. Casas, Dr. M. Á. Martínez-González, Dr. D. Corella,

Dr. J. Salas-Salvadó, Dr. J. Lapetra, Dr. E. Gómez-Gracia, Dr. F. Arós,

Dr. M. Fiol, Dr. L. Serra-Majem, Dr. E. Ros, Dr. R. Estruch

CIBER of Pathophysiology of Obesity and Nutrition (CIBEROBN)

Instituto de Salud Carlos III

Madrid 28029, Spain

The ORCID identification number(s) for the author(s) of this article can be found under https://doi.org/10.1002/mnfr.202000350

DOI: $10.1002 / \mathrm{mnfr} .202000350$
Dr. Á. Hernáez, Dr. O. Castañer, Dr. M. Fitó

Cardiovascular Risk and Nutrition Research Group

Hospital del Mar Medical Research Institute (IMIM)

Barcelona 08003, Spain

Dr. A. Tresserra-Rimbau, Dr. J. Salas-Salvadó

Unitat de Nutrició Humana, Departament de Bioquimica i Biotecnologia

Universitat Rovira i Virgili

Reus Spain

Dr. A. Tresserra-Rimbau, Dr. J. Salas-Salvadó

Hospital Universitari Sant Joan de Reus

Reus 43204, Spain

Dr. A. Tresserra-Rimbau, Dr. J. Salas-Salvadó

Institut d'Investigació Pere Virgili (IISPV)

Reus 43204, Spain

Dr. X. Pintó

Lipids and Vascular Risk Unit, Internal Medicine

Hospital Universitario de Bellvitge

L'Hospitalet de Llobregat 08907, Spain 
non-esterified fatty acids (NEFAs) and dysfunctional high-density lipoproteins (HDLs) have also been attributed pro-thrombotic properties. ${ }^{[14,15]}$ In the context of the PREDIMED (PREvención con DIeta MEDiterránea) trial, following a MedDiet decreased P-selectin levels. ${ }^{[16]}$ Beyond this study, only a smallscale prospective analysis with 21 young, healthy male volunteers indicated that following a MedDiet-like dietary pattern was related to lower fibrinogen levels and an attenuation of the coagulation response, ${ }^{[17]}$ and two cross-sectional studies have reported associations between MedDiet adherence and less D-dimer and fibrinogen values. ${ }^{[18,19]}$ However, no intervention trial has studied to date the long-term effects of this healthy dietary pattern on a comprehensive set of biomarkers of atherothrombosis.

Our main aim was to assess whether a 1-year intervention with MedDiet, enriched with virgin olive oil (MedDiet-VOO) or mixed nuts (MedDiet-Nuts), improved a set of atherothrombosis biomarkers relative to a low-fat control diet in high cardiovascular risk individuals. Our secondary aim was to determine whether 1year changes in MedDiet adherence and in the consumption of key food items of the MedDiet were associated with 1-year differences in these indicators.

Dr. M. Á. Martínez-González

Department of Preventive Medicine and Public Health

Universidad de Navarra

Pamplona 3 1009, Spain

Dr. M. Á. Martínez-González

Department of Nutrition

Harvard TH Chan School of Public Health

Boston USA

Dr. D. Corella

Department of Preventive Medicine

Universidad de Valencia

Valencia 46100, Spain

Dr. J. Lapetra

Department of Family Medicine. Research Unit.

Distrito Sanitario Atención Primaria Sevilla

Sevilla 41013, Spain

Dr. E. Gómez-Gracia

Department of Preventive Medicine and Public Health

Universidad de Málaga

Málaga 29010, Spain

Dr. F. Arós

Department of Cardiology

Hospital Universitario de Álava

Vitoria 01009, Spain

Dr. M. Fiol

Health Research Institute of the Balearic Islands (IdISBa)

Hospital Son Espases

Palma de Mallorca 07120, Spain

Dr. L. Serra-Majem

Instituto de Investigaciones Biomédicas y Sanitarias

Universidad de Las Palmas de Gran Canaria

Las Palmas 35016, Spain

Dr. E. Ros

Lipid Clinic, Endocrinology and Nutrition Service

Hospital Clínic

Barcelona 08036, Spain

Dr. R. Estruch

Internal Medicine Service

Hospital Clínic

Barcelona 08036, Spain

\section{Results}

\subsection{Study Population and Intervention}

Our study sub-sample were elderly adults (mean age 67 years, $63 \%$ women) with high prevalence of cardiovascular risk factors at baseline (78\% hypertension, 74\% hypercholesterolemia, 51\% diabetes, $50 \%$ overweight, $44 \%$ obesity, $14 \%$ current smokers, $13 \%$ antithrombotic drug users). We found no clinical differences in baseline characteristics among intervention groups (Table 1). Individuals in our analytical sample were less likely to be men and users of antithrombotic drugs, and more prone to present hypercholesterolemia than the rest of the PREDIMED study population (Table S4, Supporting Information).

Volunteers' compliance improved during the year of intervention (MedDiet-VOO: +1.14 points [95\% confidence interval: 0.79; 1.49]; MedDiet-Nuts: +1.35 [1.00; 1.69$])$. No changes in physical activity were observed (Table S5, Supporting Information).

\subsection{MedDiet Interventions and 1-Year Changes in Atherothrombosis Biomarkers}

In the whole study population, we observed significant differences between 1-year changes in the MedDiet-VOO intervention and the control diet on the activity of platelet activating factor acetylhydrolase (PAF-AH) in HDLs (adjusted difference: $+7.5 \%$ [0.17; 14.8]; Figure 1I) and HDL-bound $\alpha_{1}$-antitrypsin levels (adjusted difference: $-6.1 \%[-11.8 ;-0.29]$; Figure $1 \mathrm{H}$ ). We also detected significant differences between 1-year changes in the MedDiet-Nuts intervention and the control arm on the concentrations of NEFAs (adjusted difference: $-9.3 \%$ [-18.1; -0.53$]$; Figure 1G). Exact results are available in Table S6, Supporting Information.

In volunteers with low MedDiet adherence at enrolment, we observed a significant difference in 1-year changes in antithrombin levels between the MedDiet-VOO intervention and the control diet (adjusted difference: $+6.1 \%$ [0.76; 11.4]; Figure S1D, Supporting Information). Finally, in users of antithrombotic drugs, we also detected significant differences between the MedDiet-VOO intervention and the low-fat control arm in 1-year changes in the concentrations of D-dimer (adjusted difference: $-54.1 \%$ [-86.8; -21.3$]$; Figure S1F, Supporting Information).

When assessing post- versus pre-intervention changes, and despite these differences were not significant relative to the MedDiet interventions, the low-fat control diet was associated with increases in platelet factor- 4 concentrations $(p=0.012)$ and prothrombin fragment $1+2$ levels $(p=0.003)$ relative to baseline values (Table S7, Supporting Information).

\subsection{Cross-Sectional Associations of 1-Year Changes in MedDiet Adherence with 1-Year Differences in Atherothrombosis Biomarkers}

Substantial increments in MedDiet adherence, relative to decreases in compliance, were associated with reduced fibrinogen (adjusted difference: $-9.5 \%[-18.3 ;-0.60]$; Figure $2 B$ ) and NEFA 
Table 1. Study population.

\begin{tabular}{|c|c|c|c|c|c|}
\hline & $\begin{array}{c}\text { All } \\
(n=358)\end{array}$ & $\begin{array}{l}\text { MedDiet-VOO } \\
\quad(n=120)\end{array}$ & $\begin{array}{l}\text { MedDiet-Nuts } \\
\quad(n=119)\end{array}$ & $\begin{array}{l}\text { Low-fat diet } \\
(n=119)\end{array}$ & $p$-value \\
\hline Age [years], mean $\pm S D$ & $66.8 \pm 5.8$ & $67.3 \pm 5.3$ & $66.6 \pm 5.8$ & $66.5 \pm 6.4$ & 0.530 \\
\hline Sex (female), $n[\%]$ & $227(63.4)$ & $78(65.0)$ & $75(63.0)$ & $74(62.2)$ & 0.898 \\
\hline Type-2 diabetes mellitus, $n$ [\%] & $183(51.1)$ & $67(55.8)$ & $55(46.2)$ & $61(51.3)$ & 0.331 \\
\hline Hypercholesterolemia, $n$ [\%] & $264(73.7)$ & $88(73.3)$ & $86(72.3)$ & $90(75.6)$ & 0.834 \\
\hline Hypertension, $n[\%]$ & $278(77.7)$ & $95(79.2)$ & $92(77.3)$ & $91(76.5)$ & 0.877 \\
\hline Users of antithrombotic drugs, $n$ [\%] & $46(12.8)$ & 19 (15.8) & $13(10.9)$ & $14(11.8)$ & 0.479 \\
\hline Tobacco use: & & & & & 0.445 \\
\hline Never smoker, $n[\%]$ & $229(64.0)$ & $83(69.2)$ & $77(64.7)$ & $69(58.0)$ & \\
\hline Actual smoker, $n[\%]$ & $51(14.2)$ & $16(13.3)$ & $15(12.6)$ & $20(16.8)$ & \\
\hline Former smoker, $n[\%]$ & $78(21.8)$ & $21(17.5)$ & $27(22.7)$ & $30(25.2)$ & \\
\hline Body mass index: & & & & & 0.717 \\
\hline$<25 \mathrm{~kg} \mathrm{~m}^{-2}, n[\%]$ & $19(5.31)$ & $8(6.67)$ & $4(3.36)$ & $7(5.88)$ & \\
\hline $25.0-29.9 \mathrm{~kg} \mathrm{~m}^{-2}, n[\%]$ & $180(50.3)$ & $56(46.7)$ & $62(52.1)$ & $62(52.1)$ & \\
\hline$\geq 30.0 \mathrm{~kg} \mathrm{~m}^{-2}, n[\%]$ & $159(44.4)$ & $56(46.7)$ & $53(44.5)$ & $50(42.0)$ & \\
\hline $\begin{array}{l}\text { Mediterranean diet adherence score, } \\
\text { mean } \pm \text { SD }\end{array}$ & $8.76 \pm 1.73$ & $8.61 \pm 1.68$ & $8.69 \pm 1.85$ & $8.99 \pm 1.64$ & 0.197 \\
\hline $\begin{array}{l}\text { Leisure-time physical activity (metabolic } \\
\left.\text { equivalents of task.[min } \mathrm{w}^{-1}\right] \text { ), median } \\
\text { (1st-3rd quartile) }\end{array}$ & $1376(555-2521)$ & $1505(724-2566)$ & $1253(538-2345)$ & $1410(416-2639)$ & 0.447 \\
\hline $\begin{array}{l}\text { Alcohol intake (g per week), median (1st-3rd } \\
\text { quartile) }\end{array}$ & $15.1(0.00-80.7)$ & $12.1(0.00-53.6)$ & $30.7(0.00-94.3)$ & $10.2(0.00-79.7)$ & 0.175 \\
\hline
\end{tabular}

MedDiet-Nuts: Mediterranean diet enriched with mixed nuts; MedDiet-VOO: Mediterranean diet enriched with virgin olive oil.

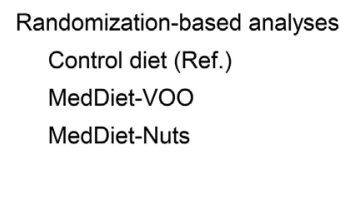

Randomization-based analyses

Control diet (Ref.)

MedDiet-VOO

MedDiet-Nuts

Randomization-based analyses

Control diet (Ref.)

MedDiet-VOO

MedDiet-Nuts

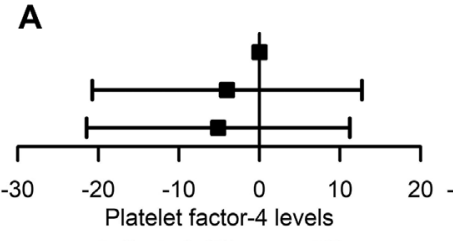

(adjusted difference, \%)

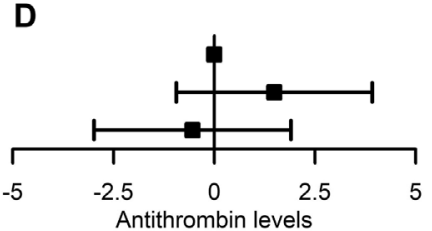

(adjusted difference, \%)

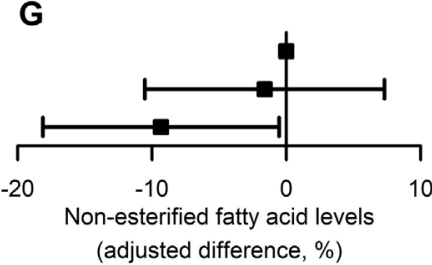

B

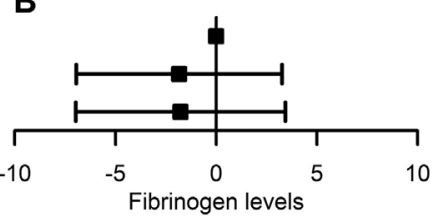

(adjusted difference, \%)

E

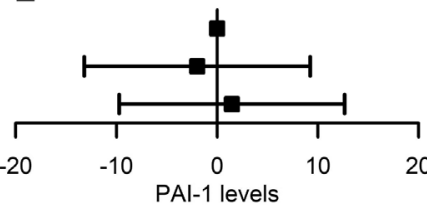

(adjusted difference, \%)
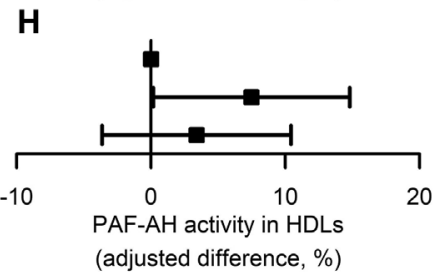

C

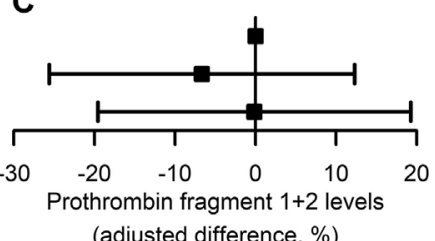

$\mathbf{F}$

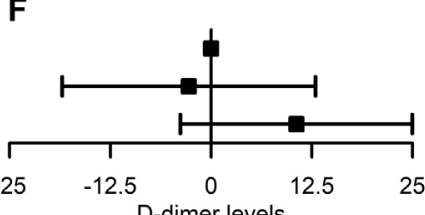

(adjusted difference, \%)

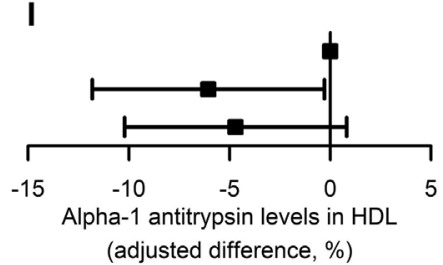

Figure 1. Differences in 1-year changes in atherothrombosis biomarkers between Mediterranean diet interventions and the control diet group (platelet factor-4 [A], fibrinogen [B], prothrombin fragment 1+2 [C], antithrombin [D], PAI-1 [E], D-dimer [F], NEFAs [G], PAF-AH activity in HDLs [H], and alpha-1 antitrypsin levels in HDL [l]). Results are shown as adjusted coefficients (percentage changes relative to baseline values) with $95 \%$ confidence intervals. HDL, high-density lipoprotein; MedDiet-Nuts, Mediterranean diet enriched with mixed nuts; MedDiet-VOO, Mediterranean diet enriched with virgin olive oil; PAF-AH, platelet activating factor acetylhydrolase; PAI-1, plasminogen activator inhibitor 1. 


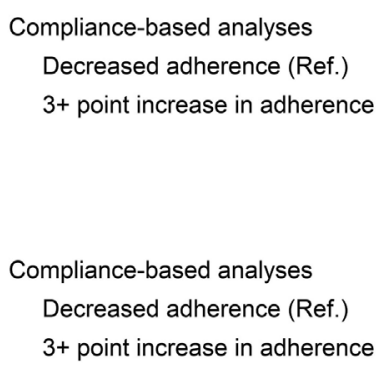

A

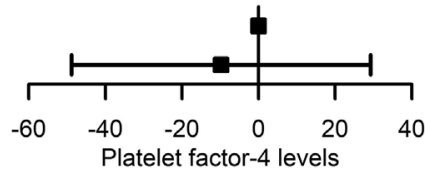

(adjusted difference, \%)

D

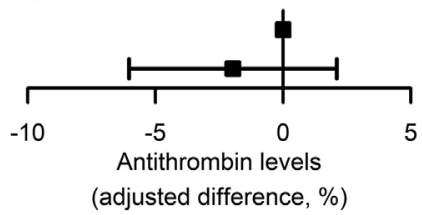

G

Compliance-based analyses

Decreased adherence (Ref.)

$3+$ point increase in adherence

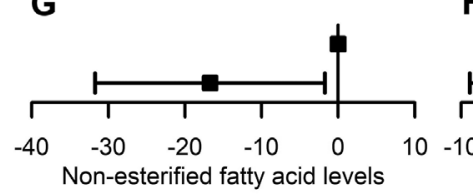

(adjusted difference, \%)
B

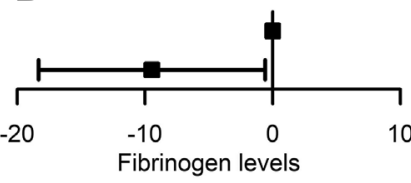

E

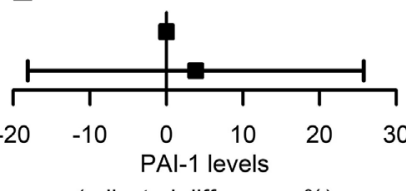

(adjusted difference, \%)

H

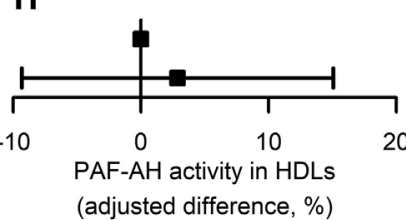

C

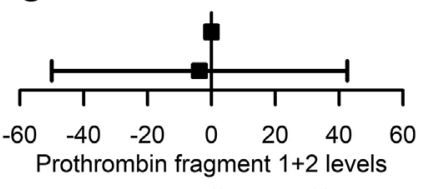

$\mathbf{F}$

(adjusted difference, \%)

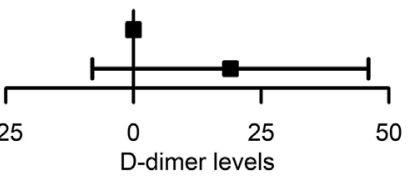

(adjusted difference, \%)

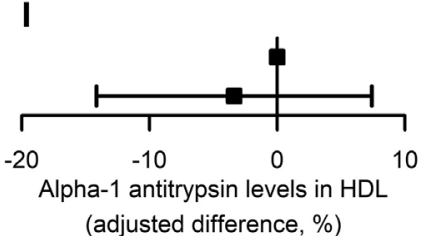

Figure 2. Associations between substantial increments in MedDiet adherence after 1 year of intervention ( $\geq 3$ score points, relative to adherence decreases) with 1-year changes in atherothrombosis biomarkers (platelet factor-4 [A], fibrinogen [B], prothrombin fragment 1+2 [C], antithrombin [D], PAI-1 [E], D-dimer [F], NEFAs [G], PAF-AH activity in HDLs [H], and alpha- 1 antitrypsin levels in HDL [I]). Results are shown as adjusted coefficients (percentage changes relative to baseline values) with $95 \%$ confidence intervals. HDL, high-density lipoprotein; PAF-AH, platelet activating factor acetylhydrolase; PAI-1, plasminogen activator inhibitor 1 .

concentrations (adjusted difference: $-16.7 \%$ [-31.7; -1.74]; Figure $2 \mathrm{G})$. Exact results are available in Table S8, Supporting Information.

\subsection{Cross-Sectional Associations of 1-Year Changes in the Intake of Key Food Items with 1-Year Differences in Atherothrombosis Biomarkers}

One-portion increments of mixed nuts were related to greater antithrombin levels (adjusted difference: $+2.2 \%$ [0.070; 4.34]). One-serving increases in the intake of fruits and vegetables were linked to lower prothrombin fragment $1+2$ (adjusted difference: $-7.6 \%[-13.1 ;-2.09])$ and platelet factor-4 concentrations, respectively (adjusted difference: $-9.3 \%[-17.6 ;-0.90]$ ). One-portion increments in fatty fish consumption were also associated with lower levels of prothrombin fragment $1+2$ levels (adjusted difference: $-13.1 \%[-26.0 ;-0.20])$. Finally, 1-serving decreases in processed meat intake were related to greater antithrombin levels (adjusted difference: $+2.0 \%$ [0.36; 3.64]), higher PAF-AH activity in HDLs (adjusted difference: $+6.1 \%$ $[1.38 ; 10.8]$ ), and lower concentrations of PAI-1 in plasma (adjusted difference: $-10.4 \%[-18.2 ;-2.71]$ ) (Table S9, Supporting Information).

\section{Discussion}

Our results show that following a MedDiet improved several atherothrombosis biomarkers in older individuals at high cardiovascular risk.

Atherothrombosis is strongly affected by inflammation and oxidative stress. ${ }^{[20,21]}$ MedDiet is a dietary pattern known to enhance these risk factors, ${ }^{[4,22]}$ possibly explaining its protective effects on thrombosis mechanisms as well. In our data, following the MedDiet-VOO intervention improved HDL antithrombotic properties (and, under certain circumstances, antithrombin and D-dimer levels), whilst the MedDiet-Nuts decreased NEFA levels. We previously reported that the MedDietVOO intervention enhanced HDL functionality in the PREDIMED Study. ${ }^{[23]}$ However, these findings extend this protection to HDL antithrombotic functions. Dietary antioxidants in the MedDiet (such as fruit- and vegetable-derived antioxidant vitamins) can bind to the structure of HDLs, contribute to their maintenance in non-oxidized forms, and help explain the reported improved activity of HDL protective enzymes. ${ }^{[24]}$ In addition, following a MedDiet has also been associated with decreased levels of pro-inflammatory molecules in circulation. ${ }^{[16]}$ This fact could contribute to explaining the reduction in the concentrations of pro-inflammatory molecules bound to HDL particles, as well as improvements in other pro-thrombotic signals (the decrease in D-dimer levels after the MedDiet intervention among users of antithrombotic drugs and the association between substantial increments in MedDiet adherence and lower concentrations of fibrinogen, both consistent with previous evidence $\left.{ }^{[18,19]}\right)$. In parallel, to explain the association between the MedDiet-Nuts intervention and lower NEFA levels, we hypothesize that polyunsaturated fatty acids (whose intake was particularly increased in this group, especially non-marine omega3 fatty acids whose intake increased by 1.15 g per day) bind to G-protein coupled receptor 120 receptors in adipose tissue, reduce lipolysis, and decrease the circulating levels of free fatty acids. ${ }^{[25]}$ The elevated consumption of dietary fiber in this dietary pattern could contribute to increasing the circulating levels of short-chain fatty acids (derived from the bacterial fermentation of fiber in the intestine), which are able to bind other Gprotein coupled receptor family receptors with a similar antilipolytic effect. ${ }^{[26]}$ 
Our study has limitations. First, our analyses were not originally included in the study protocol and, therefore, should be considered as exploratory findings. Second, study volunteers were elderly people at high cardiovascular risk, which does not allow the extrapolation of our findings to other populations. Third, we have only been able to report moderate changes or associations. However, this effect magnitude was expected because our work was based on modest real-life dietary modifications and the control diet was already a well-known healthy dietary pattern. Fourth, some of our inter-group associations (differences in 1-year changes in PAF-AH activity and $\alpha_{1}$-antitrypsin levels in apolipoprotein B-depleted plasma between the MedDiet-VOO intervention and the control arm) may rely on non-significant intragroup changes. Fifth, the statistical power of the analyses in particular sub-groups of participants could be low (Table S10, Supporting Information) and, therefore, these results should be interpreted cautiously. Sixth, cross-sectional associations of 1-year changes in the intake of key food items with 1-year differences in atherothrombosis biomarkers were not adjusted for multiple comparisons and, therefore, these findings should be considered as preliminary and verified in future studies. Finally, data on MedDiet adherence (and other covariates such as physical activity and ethanol intake) were self-reported and could be somewhat imprecise. However, the main strengths of our study were its large sample size $(n=358)$, randomized design, long-term duration (1 year), and strict quality control for laboratory biomarkers.

In conclusion, 1 year of intervention with MedDiet enhanced HDL antithrombotic properties and decreased NEFA levels in high cardiovascular risk individuals (and at a second level of relevance, increased antithrombin and reduced D-dimer concentrations in certain study subpopulations). As observed in the secondary, observational analyses, substantial increments in MedDiet adherence were associated with decreases in fibrinogen and NEFA levels. As far as we know, this is the largest, most comprehensive, hypothesis-based analysis to date of the effects of a healthy dietary pattern on atherothrombosis indicators in high cardiovascular risk individuals. Our results support the improvement of thrombosis risk status after following a MedDiet. Further studies are needed to confirm whether these improvements mediate the reported cardioprotective benefits of such lifestyle modifications.

\section{Experimental Section}

Study Design: Study subjects were participants of the PREDIMED Study. It was a large-scale, parallel, multicenter, randomized controlled trial assessing the long-term effects of following a MedDiet on the primary prevention of cardiovascular disease in high cardiovascular risk individuals. ${ }^{[1]}$ Volunteers were men (aged 55-80) and women (aged 6080) free of cardiovascular disease at enrolment but presenting type 2 diabetes mellitus or at least three of these factors: high levels of lowdensity lipoprotein cholesterol, low HDL cholesterol concentrations, hypertension, overweight/obesity, current smoking, or family history of premature heart disease. Further details of inclusion/exclusion criteria are available in Methods, Supporting Information. The trial protocol complied with the Declaration of Helsinki, was approved by local institutional ethic committees, registered under the International Standard Randomized Controlled Trial Number ISRCTN35739639 (http://www.isrctn.com/ ISRCTN35739639), and described in previous publications. [1,27] An institutional ethic committee also approved the protocol of the present sub- study. All participants provided written informed consent before joining the trial.

Individuals were randomly assigned to one of the three interventions (in a 1:1:1 ratio): 1) a MedDiet-VOO; 2) a MedDiet-Nuts; and 3) a low-fat control diet. MedDiet interventions promoted: 1) the consumption of fruits, vegetables, legumes, nuts, and fish; 2) the use of virgin olive oil as main culinary fat; 3) a decrease in the intake of ultra-processed foods/drinks and animal fats and the substitution of red/processed meats for poultry; and 4) the consumption of foods cooked by home-made methods (such as the traditional "sofrito"). To promote compliance and account for family needs, volunteers allocated in the MedDiet-VOO intervention received $1 \mathrm{~L}$ per week of virgin olive oil and those in the MedDiet-Nuts group $210 \mathrm{~g}$ per week of mixed nuts. Volunteers allocated to the low-fat control group were advised: 1) to promote the intake of fruits, vegetables, and legumes; 2 ) to decrease the consumption of high-fat dairy products, butter/margarine, meat, and ultra-processed foods/drinks, and 3) to moderate their intake of other fatty foods (olive oil, nuts, and fatty fish). Further details of the dietary intervention are available in Methods, Supporting Information.

For the present analyses, a random subsample of 358 subjects ( $4.8 \%$ of the total PREDIMED population) were selected recruited in Hospital Clinic (Barcelona) and Hospital del Mar Medical Research Institute (Barcelona) study sites, with fasting citrate plasma samples collected at baseline and after 1 year of intervention. 120 volunteers were allocated to the MedDietVOO intervention group, 119 to MedDiet-Nuts, and 119 to the control diet. Samples were stored at $-80^{\circ} \mathrm{C}$ until the analyses. The CONSORT checklist regarding the study is available in Table S1, Supporting Information.

Mediterranean Diet Adherence, Intake of Key Food Items, and Covariates: MedDiet adherence was estimated at baseline and after 1 year of intervention using the MedDiet adherence score. It was a validated short screener interrogating whether the volunteer followed 14 essential dietary characteristics associated with a MedDiet (positively scoring: intake of virgin olive oil, nuts, fruits, vegetables, legumes, fish, and wine in moderation; and the substitution or avoidance of animal fats, red and processed meats, processed foods, and sugary drinks). ${ }^{[28]}$ One-year increments in MedDiet adherence were calculated by subtracting the baseline adherence to the 1-year value.

The intake of eight key food items whose consumption was encouraged or discouraged in the MedDiet intervention (virgin olive oil, mixed nuts, fruits, vegetables, legumes, fresh fatty fish, processed meat, and alcohol) was estimated at baseline and after 1 year of intervention using a semiquantitative, validated 137-item food frequency questionnaire. ${ }^{[29]}$ One-year changes in their intake were calculated by subtracting the baseline consumption to that after 1 year of intervention, and expressed in common portion sizes $(+10 \mathrm{~g}$ per day of virgin olive oil, $+30 \mathrm{~g}$ per day of nuts, $+100 \mathrm{~g}$ per day of vegetables or fruits, $+25 \mathrm{~g}$ per day of legumes or fresh fatty fish, $-25 \mathrm{~g}$ per day of processed meat, and $+10 \mathrm{~g}$ per day of alcohol).

Data on age, sex, educational level, body mass index, smoking habit, and presence of type-2 diabetes mellitus, hypercholesterolemia, hypertension, leisure-time physical activity, and use of antithrombotic medications were gathered at baseline by trained clinical personnel. ${ }^{[1,27]}$

Atherothrombosis Biomarkers: ELISA kits were used to quantify the levels of: fibrinogen (Human Fibrinogen SimpleStep ELISA kit, ref. ab208036, abcam, UK), PAI-1 (Human PAI1 SimpleStep ELISA kit (SERPINE1), ref. ab 184863, abcam, UK), platelet factor-4 (PF4 (CXCL4) Human SimpleStep ELISA kit, ref. ab189573, abcam, UK), and prothrombin fragment 1+2 (Human F1+2 (prothrombin fragment 1+2) ELISA kit, ref. E-EL-H1793, Elabscience, USA). The concentrations of antithrombin (Antithrombin III, ref. SR-1102016, Spinreact, Spain) and D-dimer (D-Dimer, ref. SR1709231, Spinreact, Spain) were assessed by immunoturbidimetry, and of NEFAs (Non-Esterified Fatty Acids, ref. FA115, Randox, Spain) using a colorimetric technique in an ABX-Pentra 400 autoanalyzer (Horiba-ABX, France). All the previous analyses were performed in fasting citrate plasma samples. In parallel, in apolipoprotein B-depleted plasma samples (specimen in which all lipoproteins but HDLs were eliminated, also prepared from fasting citrate plasma ${ }^{[23]}$ ), total cholesterol was quantified by colorimetry (Cholesterol Enzymatic-Colorimetry, ref. SR-41022, Spinreact, Spain) and $\alpha_{1}$-antitrypsin (a-1-antitrypsin, ref. SR-1102054, 
Spinreact, Spain) by immunoturbidimetry in an ABX-Pentra 400 autoanalyzer (Horiba-ABX, France). With this data, the $\alpha_{1}$-antitrypsin/cholesterol ratio (" $\alpha_{1}$-antitrypsin levels in HDL") was calculated. The activity of the PAF-AH enzyme was determined in these samples by a colorimetric kit (PAF Acetylhydrolase Activity Assay Kit, ref. K765-1000, Cayman Chemical, USA). A detailed explanation of the quality control is available in Methods, Supporting Information and Table S2, Supporting Information.

According to the post-analysis sample sizes and standard deviations of the post- versus pre-intervention differences for each determination, the intra- and inter-group differences that were allowed to be detected with $\geq 80 \%$ power and assuming a type-I error of 0.05 (Table S3, Supporting Information) were estimated.

Statistical Analyses: The distribution of continuous variables by normality plots and the Shapiro-Wilk test was first checked. It was assessed whether there were differences in baseline values among the three intervention groups with one-way analysis of variance tests for normally distributed continuous variables, Kruskal-Wallis tests for non-normally distributed continuous ones, and chi-squared tests for categorical parameters.

It was first evaluated whether there were differences in the 1-year changes in atherothrombosis biomarkers (calculated by subtracting baseline from post-intervention values) in the MedDiet interventions relative to the control diet by multivariable linear regressions. These differences were studied in the whole population of the study (main approach), in participants with very low adherence to a MedDiet at baseline ( $<8$ score points), and users of antithrombotic drugs. ${ }^{[30]}$ It was also analyzed if there were intra-group differences between pre- and post-intervention values in every study arm by paired $t$ tests in normally distributed variables and Wilcoxon signed-rank tests in non-normally distributed ones. As secondary, observational analyses, it was studied whether substantial increments in MedDiet adherence after 1 year of intervention ( $\geq 3$ score points, in relation to decreases in MedDiet compliance), and whether 1-year changes in the consumption of key food items (virgin olive oil, mixed nuts, fruits, vegetables, legumes, fresh fatty fish, processed meat, and alcohol), were associated with 1-year differences in atherothrombosis biomarkers by multivariable linear regression models.

To help in data interpretation, the results were presented as percentage changes relative to baseline values. To calculate them, the adjusted difference coefficients obtained in the regression models were divided by the baseline value of the variable in each of the groups (in the main analyses) or in the 358 volunteers (in the secondary analyses). Models were adjusted for: age (continuous), sex, study site, educational level (primary education/secondary/higher education), baseline value of the atherothrombosis biomarker, diabetes (yes/no), hypercholesterolemia (yes/no), hypertension (yes/no), use of antithrombotic medication (yes/no), tobacco use (never/former/actual smoker), body mass index (continuous), leisuretime physical activity (continuous), alcohol use (continuous), and two propensity scores to correct for the theoretical deviations in the randomization process (calculated from 30 baseline variables). ${ }^{[1]}$ Secondary, observational analyses were additionally adjusted for the allocated intervention group and MedDiet adherence score at baseline. Models were plotted using the "Ime" package in R Software. ${ }^{[31]}$

Statistical analyses were performed with $\mathrm{R}$ software version 3.5.0.[32] The dataset analyzed during the current study is not publicly available due to national data regulations and for ethical reasons, including that there is no explicit written consent of the study volunteers to make their deidentified data available at the end of the study. However, data described in the manuscript, codebook, and analytic code will be made available upon request by sending a letter to the PREDIMED steering Committee (predimed-steering-committe@googlegroups.com). The request will be passed to all the members of the Committee for deliberation.

\section{Supporting Information}

Supporting Information is available from the Wiley Online Library or from the author.

\section{Acknowledgements}

This work was supported by grants of Instituto de Salud Carlos III [OBN17PI02, PIE14/00045_INFLAMES, CB06/03/0019, CB06/03/0028, and CD 17/00122 (A.H.)], and Agència de Gestió d'Ajuts Universitaris i de Recerca (2017 SGR 222)

The authors wish to thank Daniel Muñoz-Aguayo, Gemma Blanchart, and Sònia Gaixas for their technical assistance, and Stephanie Lonsdale for revising the English text. J.S.-S. gratefully acknowledges the financial support by ICREA under the ICREA Academia program. CIBER de Fisiopatología de la Obesidad y Nutrición (CIBEROBN) is an initiative of the Instituto de Salud Carlos III, Madrid, Spain, and financed by the European Regional Development Fund.

\section{Author Contributions}

A.H. and R.C. acquired data. A.H. analyzed and interpreted data. X.P., M.Fitó, M.A.M.-G., D.C., J.S.-S., J.L., E.G.-G., F.A., M.Fiol, L.S.-M., X.P., E.R., and R.E. conceived the clinical trial concept and design. A.H., O.C., A.T.-R., X.P., and R.E. obtained funding. A.H. drafted the manuscript. O.C., A.T.-R., X.P., M.Fitó, R.C., M.A.M.-G., D.C., J.S.-S., J.L., E.G.-G., F.A., M.Fiol, L.S.-M., X.P., E.R., and R.E. revised the content of the manuscript and approved its final version. A.H. is the guarantor of this work and, as such, had full access to all of the data in the study and takes responsibility for the integrity of the data and the accuracy of the results.

\section{Data Availability Statement}

The dataset analyzed during the current study is not publicly available due to national data regulations and for ethical reasons, including that we do not have the explicit written consent of the study volunteers to make their deidentified data available at the end of the study. However, data described in the manuscript, codebook, and analytic code will be made available upon request by sending a letter to the PREDIMED steering Committee (predimed-steering-committe@googlegroups.com). The request will be passed to all the members of the Committee for deliberation.

\section{Conflict of Interest}

X.P. reports being a board member, lecture fees, and grants from Ferrer International; being a board member and grants from the Residual Risk Reduction Initiative Foundation; personal fees from Abbott Laboratories; lecture fees and grants from Merck and Roche; lecture fees from Danone, Esteve, Menarini, Mylan, LACER, and Rubio Laboratories; and grants from Sanofi, Kowa, Unilever, Boehringer Ingelheim, and Karo Bio. J.S.-S. reports being a board member and personal fees from Instituto Danone Spain; being a board member and grants from the International Nut and Dried Fruit Foundation; personal fees from Aguas Font Vella Lanjarón, and Danone S.A; and grants from Eroski Distributors. F.A. reports personal fees from Menarini and AstraZeneca. L.S.-M. reports being a board member of the Mediterranean Diet Foundation and the Beer and Health Foundation. E.R. reports personal fees, grants, and nonfinancial support from the California Walnut Commission and Alexion; personal fees and nonfinancial support from Danone; and nonfinancial support from the International Nut Council. R.E. reports being a board member of the Research Foundation on Wine and Nutrition, the Beer and Health Foundation, and the European Foundation for Alcohol Research; personal fees from KAO Corporation; lecture fees from Instituto Cerventes, Fundacion Dieta Mediterranea, Cerveceros de España, Lilly Laboratories, AstraZeneca, and Sanofi; and grants from Novartis, Amgen, Bicentury, and Grand Fountaine. The rest of the authors have nothing to disclose.

\section{Keywords}

atherothrombosis, biomarkers, cardiovascular risk, clinical trials, Mediterranean diet 
Received: April 10, 2020

Revised: September 3, 2020

Published online: September 30, 2020

[1] R. Estruch, E. Ros, J. Salas-Salvadó, M. I. Covas, D. Corella, F. Arós, E. Gómez-Gracia, V. Ruiz-Gutiérrez, M. Fiol, J. Lapetra, R. M. LamuelaRaventos, L. Serra-Majem, X. Pintó, J. Basora, M. A. Muñoz, J. V. Sorlí, J. A. Martínez, M. Fitó, A. Gea, M. A. Hernán, M. A. MartínezGonzález, N. Engl. J. Med. 2018, 378, e34.

[2] M. A. Martínez-González, A. Gea, M. Ruiz-Canela, Circ. Res. 2019, 124, 779.

[3] D. Lairon, Mol. Nutr. Food Res. 2007, 51, 1209.

[4] M. A. Martínez-González, J. Salas-Salvadó, R. Estruch, D. Corella, M. Fitó, E. Ros, PREDIMED Investigators, Prog. Cardiovasc. Dis. 2015, 58 , 50.

[5] L. M. Ostertag, N. O'Kennedy, P. A. Kroon, G. G. Duthie, B. de Roos, Mol. Nutr. Food Res. 2010, 54, 60.

[6] J. Lopez-Miranda, J. Delgado-Lista, P. Perez-Martinez, Y. JimenezGómez, F. Fuentes, J. Ruano, C. Marin, Mol. Nutr. Food Res. 2007, 51, 1249.

[7] C. L. Wassel, C. Berardi, J. S. Pankow, N. B. Larson, P. A. Decker, N. Q. Hanson, M. Y. Tsai, M. H. Criqui, M. A. Allison, S. J. Bielinski, Atherosclerosis 2015, 239, 405.

[8] R. Altara, M. Manca, R. D. Brandao, A. Zeidan, G. W. Booz, F. A. Zouein, Clin. Sci. 2016, 130, 463.

[9] D. Feinbloom, K. A. Bauer, Arterioscler., Thromb., Vasc. Biol. 2005, 25, 2043.

[10] R. Loeffen, R. van Oerle, M. P. G. Leers, J. A. Kragten, H. Crijns, H. M. H. Spronk, H. ten Cate, PLoS One 2016, 11, e0158355.

[11] S. G. Thompson, C. Fechtrup, E. Squire, U. Heyse, G. Breithardt, J. C. van de Loo, J. Kienast, Arterioscler., Thromb., Vasc. Biol. 1996, 16, 357.

[12] M. Cortellaro, E. Cofrancesco, C. Boschetti, L. Mussoni, M. B. Donati, M. Cardillo, M. Catalano, L. Gabrielli, B. Lombardi, G. Specchia, Arterioscler., Thromb., Vasc. Biol. 1993, 13, 1412.

[13] G. D. Lowe, A. Rumley, Thromb. Haemostasis 1999, 82, 667.

[14] K. A. L. Darvall, R. C. Sam, S. H. Silverman, A. W. Bradbury, D. J. Adam, Eur. J. Vasc. Endovasc. Surg. 2007, 33, 223.

[15] M. van der Stoep, S. J. A. Korporaal, M. Van Eck, Cardiovasc. Res. 2014, 103, 362.
[16] R. Casas, E. Sacanella, M. Urpí-Sardà, G. Chiva-Blanch, E. Ros, M.-A. Martínez-González, M.-I. Covas, J. Salas-Salvadó, M. Fiol, F. Arós, R. Estruch, PLoS One 2014, 9, e 100084.

[17] D. Mezzano, F. Leighton, C. Martínez, G. Marshall, A. Cuevas, O. Castillo, O. Panes, B. Muñoz, D. D. Pérez, C. Mizón, J. Rozowski, A. San Martín, J. Pereira, Eur. J. Clin. Nutr. 2001, 55, 444.

[18] A. Di Castelnuovo, M. Bonaccio, A. De Curtis, S. Costanzo, M. Persichillo, G. de Gaetano, M. B. Donati, L. Iacoviello, MOLI-SANI Investigators, Haematologica 2017, 102, e61.

[19] S. J. Carter, M. B. Roberts, J. Salter, C. B. Eaton, Atherosclerosis 2010, $210,630$.

[20] J. E. Freedman, Arterioscler., Thromb., Vasc. Biol. 2008, 28, s11.

[21] D. D. Wagner, Arterioscler., Thromb., Vasc. Biol. 2005, 25, 1321.

[22] A. Sureda, M. d. M. Bibiloni, M. Martorell, P. Buil-Cosiales, A. Marti, A. Pons, J. A. Tur, M. Á. Martinez-Gonzalez, Mol. Nutr. Food Res. 2016, 60, 2654.

[23] A. Hernaez, O. Castañer, R. Elosua, X. Pinto, R. Estruch, J. SalasSalvado, D. Corella, F. Aros, L. Serra-Majem, M. Fiol, M. OrtegaCalvo, E. Ros, M. A. Martinez-Gonzalez, R. de la Torre, M. C. LopezSabater, M. Fito, Circulation 2017, 135, 633.

[24] A. Hernáez, M. Farràs, M. Fitó, Curr. Opin. Lipidol. 2016, 27, 47.

[25] D. Y. Oh, S. Talukdar, E. J. Bae, T. Imamura, H. Morinaga, W. Fan, P. Li, W. J. Lu, S. M. Watkins, J. M. Olefsky, Cell 2010, 142, 687.

[26] G. den Besten, K. van Eunen, A. K. Groen, K. Venema, D.-J. Reijngoud, B. M. Bakker, J. Lipid Res. 2013, 54, 2325.

[27] M. A. Martinez-Gonzalez, D. Corella, J. Salas-Salvado, E. Ros, M. I. Covas, M. Fiol, J. Warnberg, F. Aros, V. Ruiz-Gutierrez, R. M. LamuelaRaventos, J. Lapetra, M. A. Munoz, J. A. Martinez, G. Saez, L. SerraMajem, X. Pinto, M. T. Mitjavila, J. A. Tur, M. P. Portillo, R. Estruch, Int. J. Epidemiol. 2012, 41, 377.

[28] H. Schröder, M. Fitó, R. Estruch, M. A. Martínez-González, D. Corella, J. Salas-Salvadó, R. Lamuela-Raventós, E. Ros, I. Salaverría, M. Fiol, J. Lapetra, E. Vinyoles, E. Gómez-Gracia, C. Lahoz, L. Serra-Majem, X. Pintó, V. Ruiz-Gutierrez, M. Covas, J. Nutr. 2011, 141, 1140.

[29] J. D. Fernández-Ballart, J. L. Piñol, I. Zazpe, D. Corella, P. Carrasco, E. Toledo, M. Perez-Bauer, M. Á. Martínez-González, J. Salas-Salvadó, J. M. Martn-Moreno, Br. J. Nutr. 2010, 103, 1808.

[30] J. F. Viles-Gonzalez, V. Fuster, J. J. Badimon, Eur. Heart J. 2004, 25, 1197.

[31] D. Bates, M. Mächler, B. Bolker, S. Walker, J. Stat. Softw. 2015, 67, 1.

[32] R Core Team, 2014. 